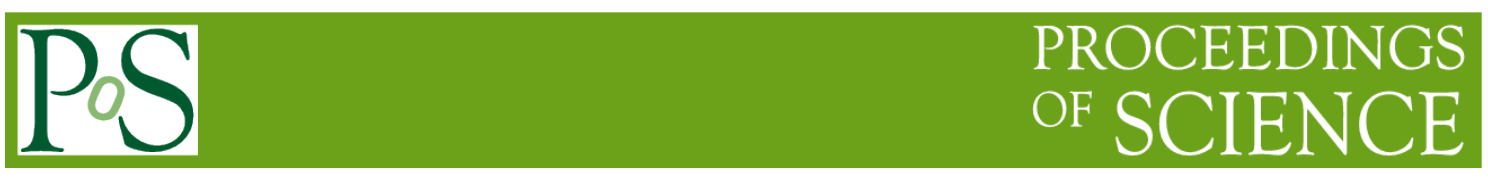

\title{
Status of e-Science Activities in Mongolia (Heritage Science)
}

\section{Nergui B. ${ }^{1}$}

Department of Informatics, Institute of Physics and Technology of Mongolian Academy of Sciences Enkhtaivan ave, 54b Ulaanbaatar 13330, Mongolia

E-mail: abnerguiearvis.ac.mn

\section{Batzaya E.}

Department of Informatics, Institute of Physics and Technology of Mongolian Academy of Sciences Enkhtaivan ave, 54b Ulaanbaatar 13330, Mongolia

E-mail: zaya_alternative@yahoo.com

Saving cultural heritages have been always an important matter for Mongolia therefore in the Mongolian Academy of Sciences have experience and collaboration with other research institutes, this paper covers the projects which are delicate to save our cultural treasures. In order to save it there is an issue, which is how to store cultural heritages and MAS working on the digitization projects that is an extremely good way to store the cultural heritages.

International Symposium on Grids and Clouds 2016

13-18 March 2016

Academia Sinica, Taipei, Taiwan

\section{${ }^{1}$ Speaker}




\section{Introduction}

Unlike different universities in Mongolia, the institutes and centers of the MAS have much information about history, archaeology, paleontology, geography, botany, biology, natural resources, language and literature of Mongolia which has been collected due to the research activities and field investigation performed for many years. In order to have a public access to this information, it is vitally important to create an integrated computerized database and disseminate the information via the Internet and electron publishing.

For example:

- There are about 10,000 scientific base documents,

- catalog including description of 7,800 species of insects,

- catalog of habitat description of 9,000 insects,

- 4,000 stuff models of 4,000 birds.

- Mongolian state fund and private herbarium collections possess 75,000 perennial, 3,500 vegetation species of swamp areas, 10,000 weed plants, 10,000 moss plants and 2,500 fungus species.

- $\sim 600$ traditional songs and other folklore, $\sim 300$ art works, 20 sculptures and more than 40 kinds of instruments.

Example research studies include:

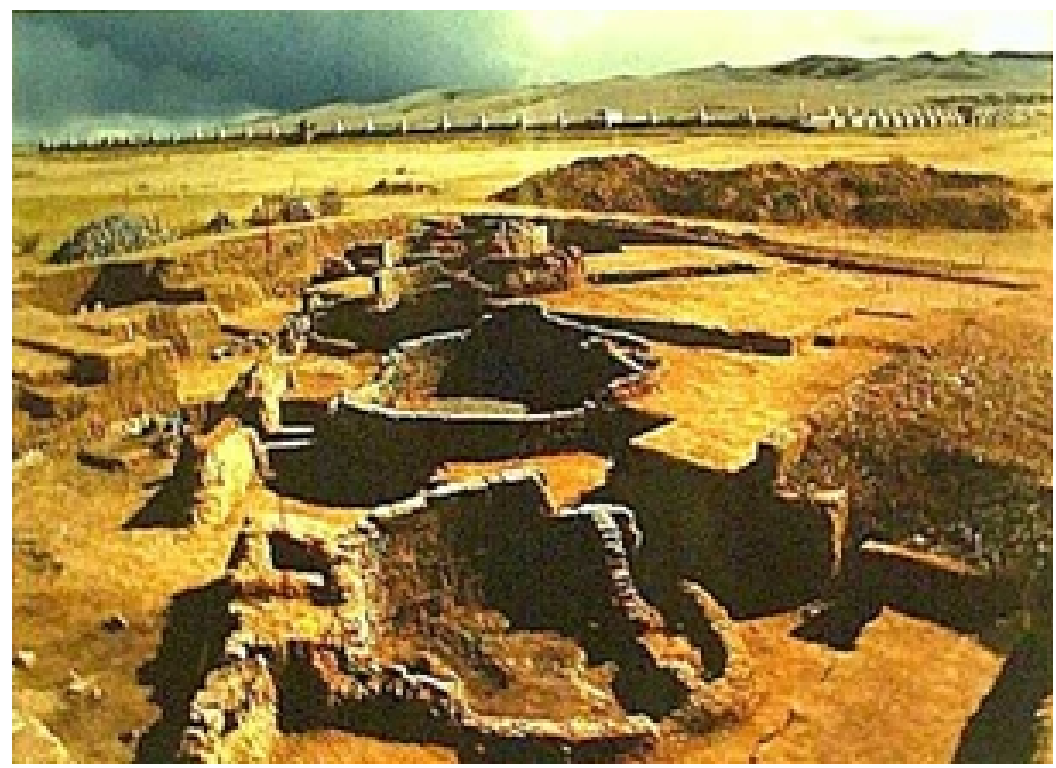

Figure.1 Archaelogical investigation of ancient capital of Mongolian Empire KharaKhorim. 


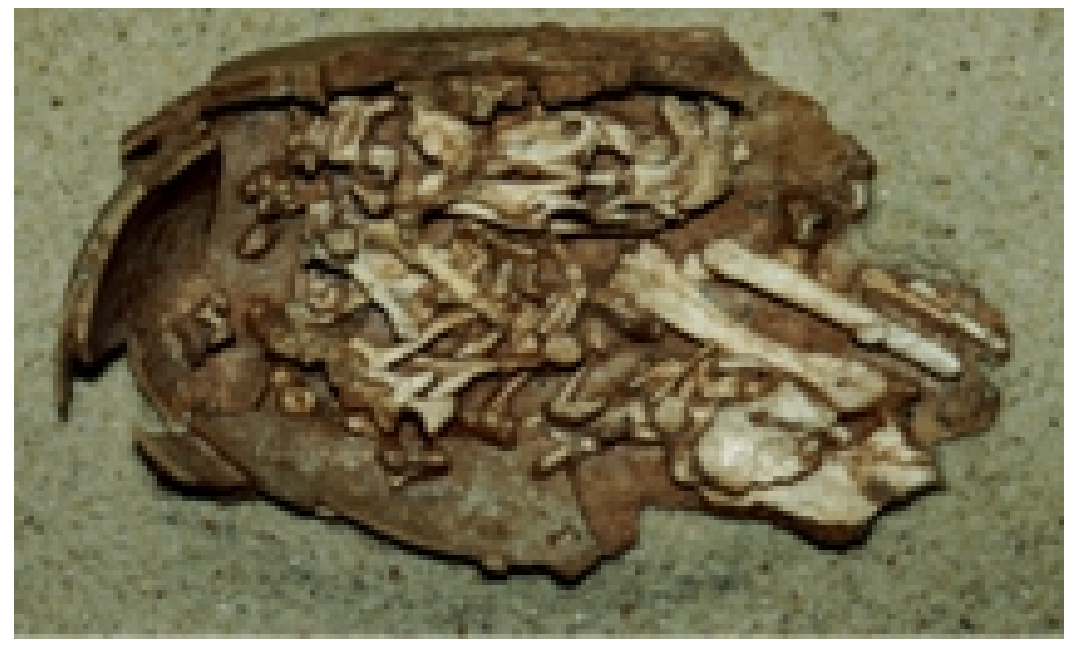

Figure.2 The Oviratorid embryo from Ukhaa Tolgod, Mongolia, in the Gobi desert is the first definitive embryo of nonavian theropod dinosaur. This embryonic skelet on of a nonavian theropod dinasour was found preserved in an egg from Upper Cretaceous rocks in the Gobi desert of Mongolia in 1993. The egg containing the embryo is incomplete, but the preserved portion is similar to oblong eggsclassified as elongatodithid.

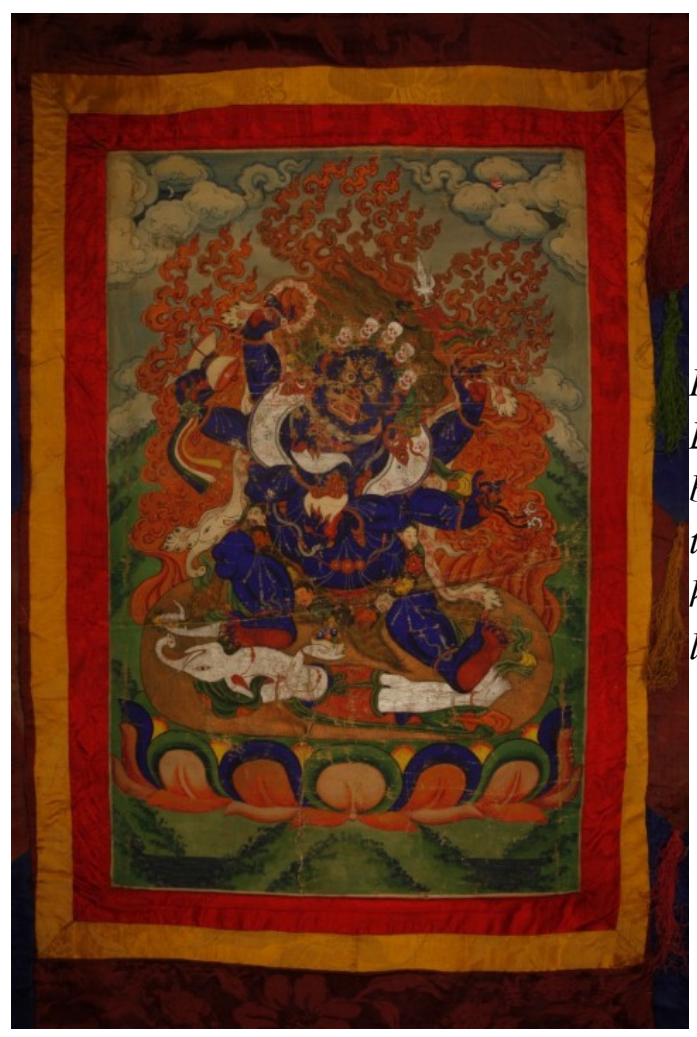

Figure. 3 this is the painting (Tanga) of Ochirvan Buddha who protects all Mongols. Chinggis han is believed to be his spirit. There is a hypothesis that this painting might have been created under Bogdo khan's decree in around 1911 after Mongolia was liberated from the Manchu oppression. 


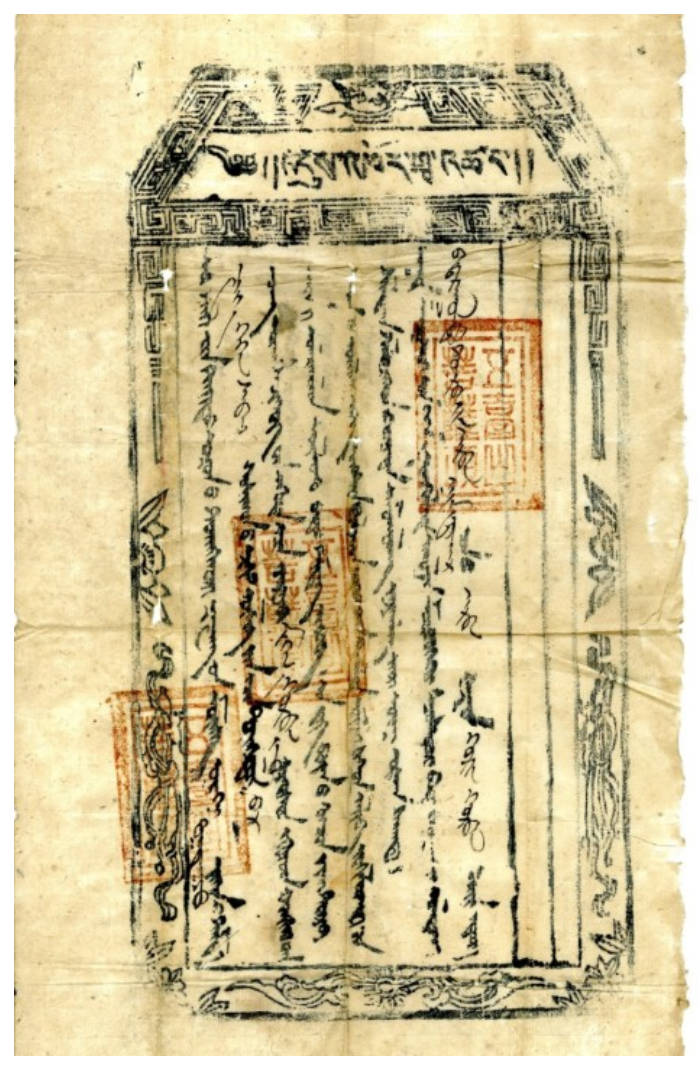

Figure.4 All over Mongolia charity contributions from the Tibetan temples and monasteries were collected every year, and the contributors were granted with so called "Badar temdeg" papers/credentials. Those papers have been kept in individual families from generation to generation.

\subsection{Cultural Heritage Digitization Projects in Mongolia}

Mongolian people have a rich long history of written and verbal culture and a specific in the form of petroglyphs, birch-bark and paper printed sutras along with unique art works by craftsmen and embroiderers are being kept in the National Library, Gandan Tegchinlen monastery and the Institute of Language and Literature of the Academy of Sciences and museums.

Academician Ts. Damdinsuren, B. Rinchen, D. Altangerel, Dr. D. Yondon and Dr. L. Khurelbaatar founded the library by collecting numerous sutras in Mongolian script, literature and history during their scientific expeditions around the country since 1960s. In recent years, an archeological research team led by Prof. A. Ochir from the International Nomadic Civilization Institute collected manuscripts written on the birch bark.

Today, books and manuscripts are on the verge of destruction as paper dries out and burns due to the storage mishandling. Moreover, study of rare script monuments and intellectual property, their availability to and use by the public should be addressed immediately. One of solutions could lie in digitizing the ancient sutras. The good news is that there have been some positive steps taken lately with assistance from the Government and international organizations.

Today, there are three projects taking place in order to implement digitizing our cultural heritage:

The First one is the joint preservation project between the National Library of Mongolia and Asian Classics Input Project. Its aim is to digitize the library collection of over 1 million sutras and manuscripts. Within the scope of this project the joint team has produced a digital 
catalog of sutras that includes biographies and praises, Buddhist philosophy and knowledge printed on the wooden birch bark and written by Holy lamas such as Bogda Zonhawa, Khaidubje, Jaltsabje and Dalai Lama. The digital catalog now contains 86,140 sutras equal to 18.6 percent of the total aim.

The National library in the partnership with the Digital Preservation Society started distributing the digital version of Tempangma manuscript and the Peking edition of the Kangyur from the permanent collection of the National Library of Mongolia.

In 2005, the "Digitization project for the national cultural heritage information" was approved by the Government of Mongolia. The project will be carried out until 2015 and will involve 35 organizations, including scientific research institutes, national and regional museums. In the framework of the project, the Center of Cultural Heritage has been executing the registration and documentation of historical and cultural immovable properties, artifacts, archaeological and paleontological findings to establish the national cultural heritage information fund with collected data. As of the end of 2010, the project has registered 26,438 digitized artifacts.

Despite these efforts to register and preserve cultural heritage, there has been little else done in terms of scientific collaborations. Without the proven technology for implementing large-scale digital repositories there aren't any cultural heritage collections available online.

Addressing scientific and technological issues in cultural heritage, the Mongolian Academy of Sciences, the leading research organization for fundamental research, is aiming at an innovative infrastructure for scientific research, collaboration and distribution of the cultural heritage to public. In November 2010, in the framework of scholarly cooperation between the Mongolian Academy of Sciences and the Max Planck Society a competence center for digitization of Mongolian cultural heritage was founded in Ulaanbaatar.

\subsubsection{Competence centre for Digitization of Cultural Heritage of the Mongolian Academy of Sciences}

This center is working to utilize progressive technological methods that will digitize cultural heritage items, make cultural heritage accessible to the public with researcher's explanations and build a digital information system that will facilitate scientific collaboration. The Mongolian Academy of Sciences consists of 17 research institutes, 9 innovation centers, ranging from archaeology and paleontology to cosmology and information technology. The main difference between the competence center for digitization of cultural heritage and other centers is that it doesn't intend to make mass digitization for some specific libraries. This center aims to digitize manuscripts and books which contain traditional knowledge and technology and distribute them to the public. We also aim to collaborate with the ECHO system and introduce it into Mongolian practice. This Center has a digital camera CANON EOS5D Mark II and other equipment provided by the Max Planck Society. For the first 12 months since its establishment the Center has digitized about 12 manuscripts and paid them in ECHO system.

Over 40 books and sutras of Tibetan works, traditional medicine, Mongolian craftsmanship and other kinds have been digitized. In collaboration with the Institute of Language and Literature, the Center will work on digitization and cataloging about 1,100 monuments of Clear Script, over 1,000 of Mongolian and nearly 8,000 Tibetan books that were accumulated at the Academy of Sciences. 
The international Institute of Nomadic Civilization has forwarded a proposal to carry out research based on this Center on a comparative study of manuscripts and books printed on the birch bark, that were widely used among the $16-17^{\text {th }}$ century Mongolian nomads with other birch-bark art monuments in respect of their methods, technology, customs and distribution, identification of peculiar and common features of the birch-bark culture of the Mongolian nomads, digitization and cataloging of manuscripts, providing their availability to the public, putting it to research use, and its preservation.

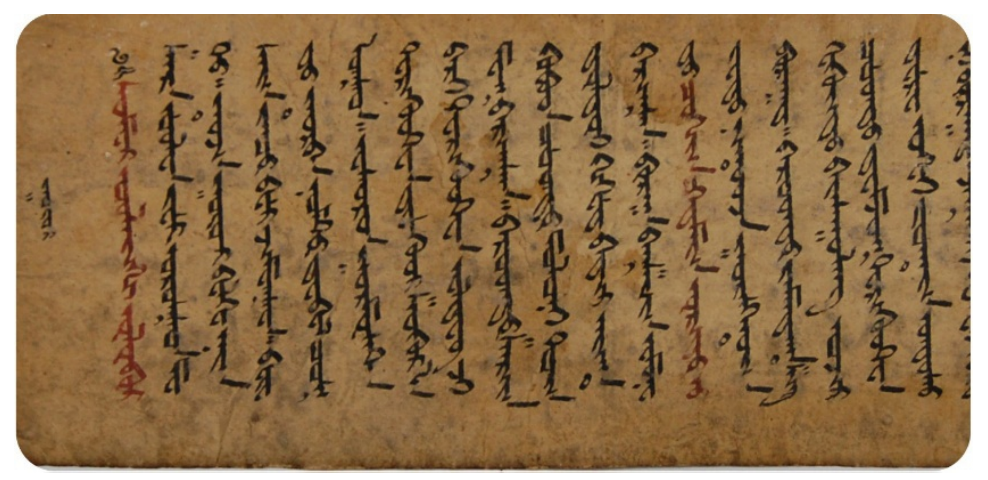

Figure.7 1150 sutras stored in the Clear script have been digitalized.

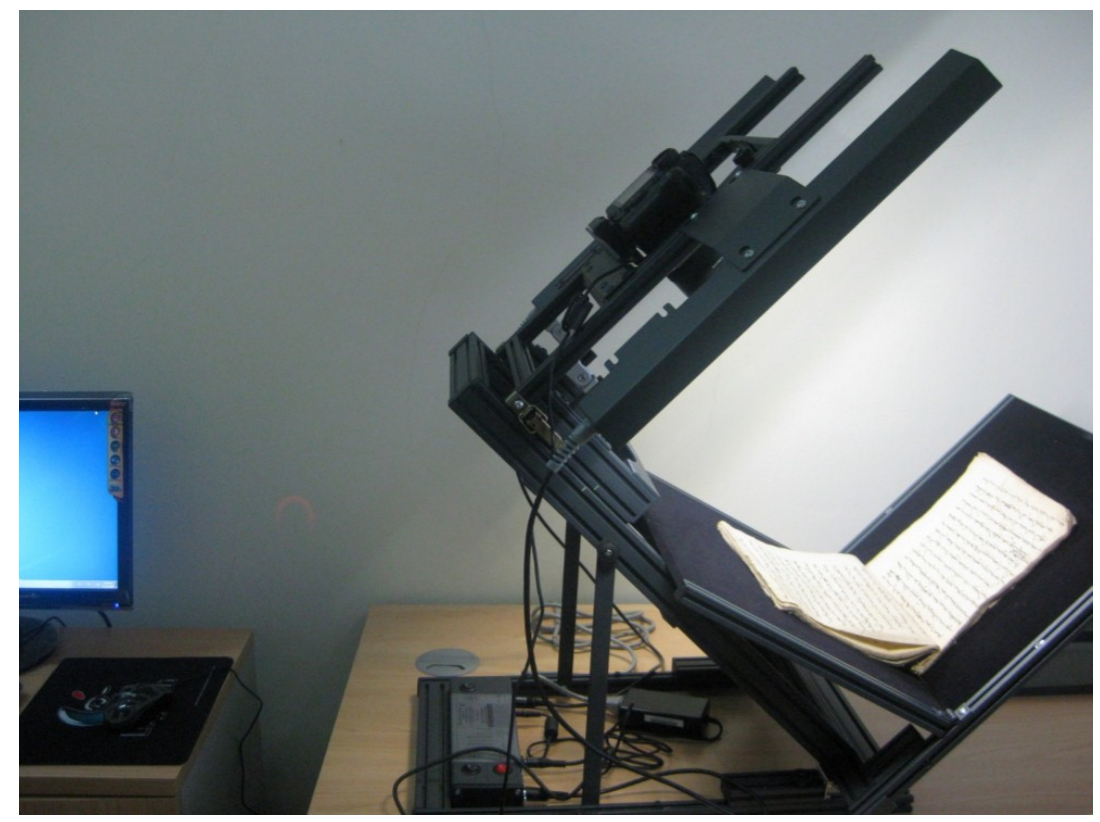

Figure.8 Digital camera CANON EOS5D Mark II and other equipment provided by the Max Planck Society.

In regard of protecting heritage of Mongolian books and sutras, I would like to forward the following ideas:

\subsubsection{Conclusion}

To organize immediately the equipping of book storage with designated equipment in order to improve protection and preservation of books. For this, do design the equipment domestically and use it instead of expecting financial possibilities to purchase expensive foreign 
equipment. I think it is feasible to use directly result of research on adjusting a regular regime of automatic close environmental work, accumulated at the Academy. Also digitize damaged books, using delicate technology and introduce advanced methods, The 2015 - 2018 project "The Innovative Application of Multi-Spectral Technology for Culture Heritage" has successfully launched as a result of expanding cooperation with the SINICA Academy of Sciences of Taiwan since 2012. The participants of this project are: Informatics Sector/Division of the Physics and Technology Institute of the Mongolian Academy of Sciences, the SINICA Academy of Sciences of Taiwan, Chinese Culture University, and Tamkang University.

References

[1] http://docslide.us/documents/pnc-2010-annual-conference-dec-1-3-2010-hong-kong.html

[2] http://pos.sissa.it/archive/conferences/153/042/ISGC\%202012_042.pdf

[3] http://www.pnclink.org/pnc2012/english/program.html 